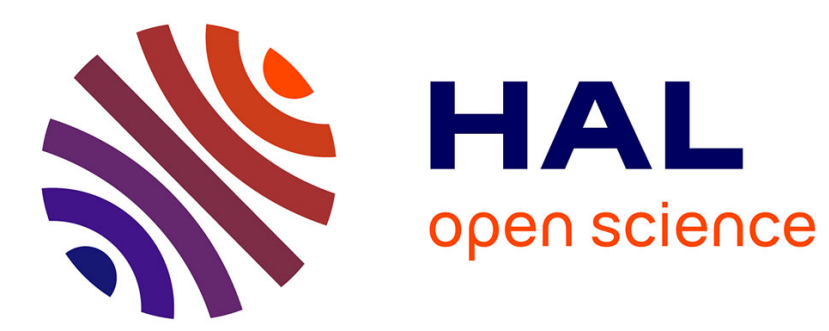

\title{
Conditional Risk-Based Portfolio
}

Olessia Caillé, Daria Onori

\section{- To cite this version:}

Olessia Caillé, Daria Onori. Conditional Risk-Based Portfolio. 2018. hal-01973115

\section{HAL Id: hal-01973115 \\ https://hal.science/hal-01973115}

Preprint submitted on 8 Jan 2019

HAL is a multi-disciplinary open access archive for the deposit and dissemination of scientific research documents, whether they are published or not. The documents may come from teaching and research institutions in France or abroad, or from public or private research centers.
L'archive ouverte pluridisciplinaire HAL, est destinée au dépôt et à la diffusion de documents scientifiques de niveau recherche, publiés ou non, émanant des établissements d'enseignement et de recherche français ou étrangers, des laboratoires publics ou privés. 
Document de Recherche du Laboratoire d'Économie d'Orléans

Working Paper Series, Economic Research Department of the University of Orléans (LEO), France

DR LEO 2018-13

\section{Conditional Risk-Based Portfolio}

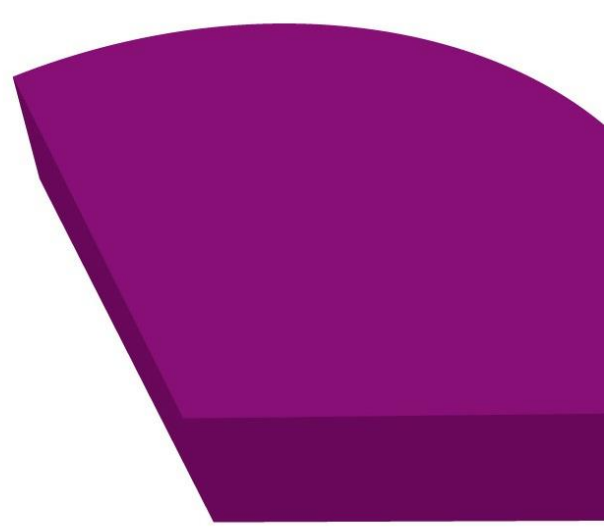

Olessia CAILLÉ

Daria ONORI

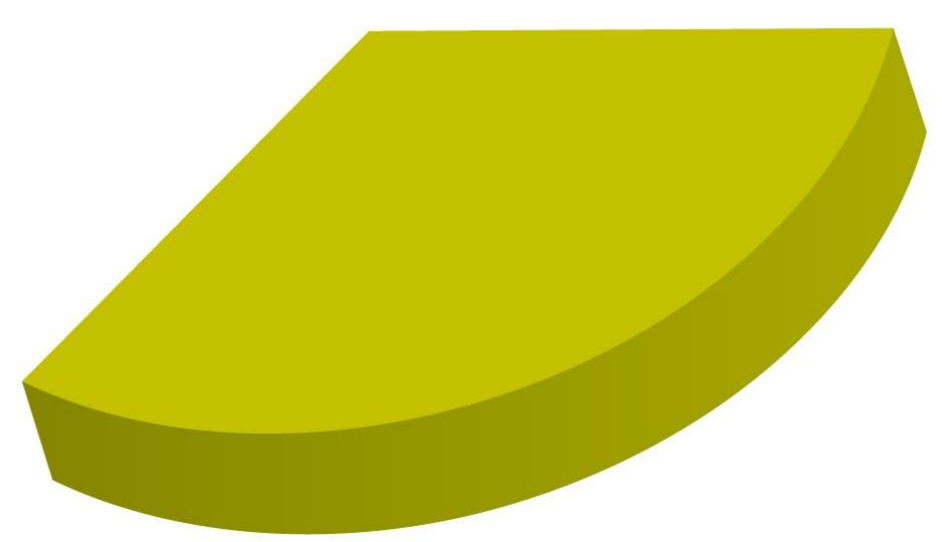

Mise en ligne / Online : 26/11/2018 


\title{
Conditional Risk-Based Portfolio*
}

\author{
Olessia Caillé ${ }^{\dagger} \quad$ Daria Onori ${ }^{\ddagger}$
}

November 9, 2018

\begin{abstract}
Risk-based investment strategies such as Minimum Variance, Maximum Diversification, Equal Risk Contribution, Risk Parity, etc. share the common feature of being based on a risk measure, typically the covariance matrix of the asset returns. When one comes to implement these strategies, the usual approach consists in using an unconditional covariance matrix, simply estimated by the sample covariance matrix of past returns over a rolling window. An alternative consists in using a conditional covariance matrix computed from a multivariate GARCH-type model and which depends on information available to date. In this paper, we propose the first unifying and systematic comparison framework for the unconditional and conditional risk-based investment strategies. We compare their out-of-sample performances in terms of risk, returns and turnover (trading volume) with 4 criteria across 3 empirical datasets. Our results show that conditional risk-based strategies do not improve the out-ofsample Sharpe ratios as well as the ex-post risk, but logically increase the turnover.
\end{abstract}

Keywords: Risk-based investment strategies, Conditional risk measures, Minimum Variance, Maximum Diversification, Equal Risk Contribution, Risk Parity

JEL classification: G10, G11.

\footnotetext{
*We would like to thank Ophélie Couperier, Christophe Hurlin, Jérémy Leymarie, Louis Raffestin and the participants to the Young French Econometricians seminars for their comments. We thank the ANR MultiRisk (ANR-16-CE26-0015-01) for supporting our research.

${ }^{\dagger}$ University of Orléans, CNRS, LEO, FRE 2014, F45067, Orléans, France. Corresponding author: olessia.caille@univ-orleans.fr.

${ }^{\ddagger}$ University of Orléans, CNRS, LEO, FRE 2014, F45067, Orléans, France. email: daria.onori@univ-orleans.fr.
} 


\section{Introduction}

Risk-based portfolio strategies such as Minimum Variance (MV), Maximum Diversification (MD), Equal Risk Contribution (ERC), Risk Parity (RP), etc., are largely used by the asset management industry within many popular investment vehicles (smart beta ETF, mutual fund, etc.). Jurczenko et al. (2013) mention some of these uses in multi-asset allocation for more robustness in strategic decisions; in equities as alternatives to market capitalization benchmarks, which are heavily concentrated in a few stocks and significantly biased towards overvalued stocks and sectors; in the smart beta exchange-traded funds (ETFs) industry, and so on. Considering only RP strategies, the asset under management (AUM) of related investments is estimated at $\$ 400 \mathrm{Bn}$ (Financial Times, 2015), and in a recent survey of quantitative investment managers (800 clients of JPM in US and Europe), Kolanovic et al. (2015) found that 50\% prefer a Risk Parity approach, versus 15\% for traditional fixed weights, 20\% Markowitz mean-variance optimization, and $20 \%$ active asset timing. ${ }^{1}$

Such a success is largely explained by the main common feature of these strategies. Whatever their definition and objective, the risk-based investment strategies do not require to forecast the expected returns and only rely on the estimation of a risk measure (volatility, Value-at-Risk, etc.) for the portfolio returns. Indeed, it is well-known that the traditional mean-variance optimization turns out to be an "estimation-error maximization" (Michaud, 1989). In particular, it relies on the estimation of expected returns which are notoriously unstable and hard to predict (Merton, 1980). Furthermore, the mean-variance portfolios are extremely sensitive to the estimation errors in means (Frankfurter et al., 1971; Chopra and Ziemba, 1993; Kan and Zhou, 2007). In contrast, risk-based investment strategies only require the estimation of risk and dependencies between assets, generally through the covariance matrix of asset returns. This is for instance the case for the MV portfolio, i.e. the portfolio with smallest feasible variance which can be constructed from the available securities. Similarly, the covariance matrix is the only required input to derive the MD allocation which maximizes the ratio between undiversified and diversified volatility of the portfolio (Choueifaty and Coignard, 2008). It is also the case for the ERC and RP strategies (Maillard et al., 2010) which define a different approach to diversification, by spreading the ex-ante total risk equally among the portfolio components. ${ }^{2}$

In practice, all risk-based strategies require an estimate of the covariance matrix $\Sigma$, at each rebalancing date. Asset managers and academics generally consider the unconditional covariance

\footnotetext{
${ }^{1}$ Kolanovic et al. (2015) mention a total AUM for the RP strategies equal to $\$ 500 \mathrm{Bn}(40 \%$ of these assets are allocated to equities).

${ }^{2}$ The RP strategy (Qian, 2005; Roncalli, 2013) can be viewed as a generalization of the ERC in which the risk contributions are set equal to a risk budgeting target, not necessarily equal to $1 / n$.
} 
matrix, the estimator of which is simply defined by the sample covariance matrix of past returns over a rolling window. However, an alternative consists in using a conditional covariance matrix that depends on information available to date and a specific model, typically a multivariate GARCH-type model.

This paper provides the first systematic comparison of unconditional and conditional riskbased investment strategies. We evaluate the out-of-sample relative performance of these strategies, in terms of ex-post returns, ex-post risk, and portfolio turnover. Both unconditional and conditional approaches have pros and cons. The unconditional approach has the great advantage of being model free and simple to implement, since no structure is imposed on the covariance matrix. However, it suffers from various drawbacks which have been largely documented in the literature devoted to mean-variance optimization. First, it implies to assume that the asset returns are stationary and $\Sigma$ is constant over time. Second, the sample covariance matrix is a reliable estimator as long as the sample size is much greater than the number of assets. If this condition is not fulfilled, robustification methods are necessary (see for instance Ledoit and Wolf (2004) and Ledoit and Wolf (2012), among others). Finally, as noticed by Martellini et al. (2014), the estimate depends on a particular historical scenario, and may not be fully representative of the true distribution of returns. In particular, the size of the estimation window plays a crucial role: increasing the window size diminishes the weight of recent information, and makes the covariance estimates less reactive to new information. At the limit, they are time-invariant. On the contrary, a small estimation window may induce estimation problems, as the covariance estimates are less robust, especially when the number of assets is large.

Conversely, the conditional approach implies to specify a model, typically a multivariate GARCH-type model, for the dynamics of the conditional covariance matrix (see Bauwens et al. (2006) for a survey). The variance forecasts, and ultimately the optimal asset allocations, are likely to be affected by potential model misspecification and estimation errors. ${ }^{3}$ However, conditional risk-based strategies have some advantages. The portfolios are likely to be more reactive as covariance estimates immediately incorporate new information. Thus, we could expect that they imply less ex-post risk than corresponding unconditional portfolios, even if the turnover (trading volume) is likely to be higher. The potential gains in terms of return performances of the conditional approach are a priori unclear.

Here, we propose a systematic and unifying comparison framework for the 3 most popular risk-based investment strategies, namely MV, ERC, and MD. For each of them, we compare the

\footnotetext{
${ }^{3}$ Ardia et al. (2017) recently propose an evaluation, based on Monte Carlo simulations, of the impact of covariance misspecification in risk-based portfolios.
} 
out-of-sample performance of the unconditional and conditional risk-based optimal portfolios, using the 4 standard performance criteria: (1) the out-of-sample Sharpe ratio, (2) the ex-post return-losses, (3) the turnover, and (4) the ex-post portfolio volatility. We also consider the equally weighted portfolio as a benchmark, as in DeMiguel et al. (2009). The conditional riskbased allocations are computed from the out-of-sample covariance matrix forecasts obtained from a DCC-GARCH model (Engle, 2002), which is considered as a benchmark among the MGARCH models. In what follows we refer to this model as DCC for sake of simplicity. Applying a rollingwindow estimation procedure, both for the DCC parameters and the sample covariance matrix of past returns, we consider estimation windows of different lengths (500 or 1,000 observations, respectively) and different forecasts horizons. The forecast horizons are determined by the portfolio rebalancing frequencies, which are fixed at 1 day, 1 week or 1 month. We apply our comparison procedure on 3 datasets which have been used in the seminal paper of DeMiguel et al. (2009) in their seminal paper, with additional data to the end of 2016. It is well-known that the conditional heteroskedasticity (ARCH effect) depends on the return sampling frequency (intraday, daily, weekly, monthly, etc.). Here, we use daily returns for the estimation procedure as it is the most favorable for conditional heteroskedasticity, and ultimately for the conditional approach.

Even within such a favorable framework, our empirical results are mitigated for the conditional approach. First, conditional risk-based strategies do not improve the out-of-sample Sharpe ratios obtained with basic unconditional approaches. We only observe that increasing the estimation windows' length tends to deteriorate the performance of unconditional portfolios, and as consequence to improve the relative performances of conditional approaches. The rebalancing frequency has no clear-cut influence on our diagnostic. Second, as expected, the turnovers are higher for the conditional strategies than for the corresponding unconditional ones. Third, the ex-post risk is not reduced by the conditional approach as expected. These results are robust when we (i) distinguish between crisis and calm estimation periods, and (ii) consider alternative multivariate GARCH models.

Our paper contributes to the literature devoted to risk-based investments, and especially to the literature dealing with the issue of their historical performance (Chow et al. (2011), Leote de Carvalho et al. (2012), Choueifaty et al. (2013), among others). These studies are generally based on a comparison of the risk-based portfolio returns, with the returns of various alternative portfolios, including market-capitalization portfolios. However, these historical assessments only rely on unconditional approaches. To the best of our knowledge, Martellini et al. (2014) is the only study that proposes a conditional strategy for risk-based investment. The authors introduce 
three distinct conditional RP strategies, explicitly designed to optimally respond to changes in state variables that have been used in the literature as proxies for the stochastically time-varying opportunity set. These strategies are based on three downside risk measures, namely the semivariance, Value-at-Risk, and Expected Shortfall. They conclude to the superiority in various economic regimes of such conditional RP strategies with respect to standard RP techniques based on unconditional volatility. Their goal and methodological approach is different from ours. First, they only consider the RP strategy and not the other risk-based investment strategies. Second, they propose new RP conditional strategies, based on downside risk measures. Our goal is different and consists in assessing the gains associated to the use of a conditional covariance matrix for each of the risk-based investments strategies. The negative results that we found in this paper have direct implications for the asset management industry (see Harvey (2017) for a general discussion about negative results in finance). They confirm the merits of the standard approach based on unconditional risk measures, currently used by practitioners, and its performances in terms of returns and turnover.

The rest of the paper is organized as follows. In Section 2, we present the risk-based strategies. In Section 3, we detail the implementation of the unconditional and conditional approaches, and the estimation methodology. We also detail our unifying comparison framework and the criteria used to assess the out-of-sample performance of the portfolios. In Section 4, we describe the datasets. In Section 5, we conduct our empirical analysis and display our main results. Section 6 analyses the robustness of our findings. We summarize and conclude our paper in Section 7.

\section{Risk based strategies}

In this section, we present the main risk-based investment strategies and introduce the distinction between conditional and unconditional risk-based allocations. Consider a universe of $n$ assets and denote by $r_{t}=\left(r_{1 t}, \ldots, r_{n t}\right)^{\top}$ the $n$-dimensional vector of returns at time $t$. Denote by $\omega=\left(\omega_{1}, \ldots, \omega_{n}\right)^{\top}$ the $n$-dimensional vector of portfolio weights. For sake of simplicity, we impose no short-selling for all the strategies, meaning that $\omega \geq 0$. The sum of the weights is equal to 1, i.e. $e^{\top} \omega=1$ with $e$ the unit vector. Denote by $\sigma_{i}^{2}$ the variance of asset's return $i$ and by $\sigma_{i j}$ the covariance between asset $i$ and $j$ for $i \neq j$. Finally, let $\Sigma$ be the covariance matrix of $r_{t}$ and $\sigma_{p}=\left(\omega^{\top} \Sigma \omega\right)^{1 / 2}$ the standard deviation (volatility) of the portfolio returns.

Following Jurczenko et al. (2013), we define a risk-based portfolio as the allocation $\omega^{*}$ sat- 
isfying the following optimization program:

$$
\begin{array}{r}
\omega^{*}=\underset{\omega}{\arg \min } D\left(f\left(\omega_{i} ; \Sigma, \gamma, \delta\right)\right) \\
\text { u.c. }\left\{\begin{array}{c}
\sum_{i=1}^{n} \omega_{i}=1 \\
\omega_{i} \geq 0
\end{array}\right.
\end{array}
$$

where $f\left(\omega_{i} ; \Sigma, \gamma, \delta\right)=\frac{\omega_{i}^{\gamma}}{\sigma_{i}^{\delta}} \times \frac{\partial \sigma_{p}}{\partial \omega_{i}}$, with $\gamma \geq 0$ and $\delta \geq 0$ two parameters, and $D($.$) a dispersion$ metric such as the standard-deviation or the mean absolute deviation. The term $\partial \sigma_{p} / \partial \omega_{i}$ represents the marginal risk contribution of the $i^{t h}$ asset, which corresponds to the sensitivity of the portfolio volatility to a small change in the weight of asset $i$. Hence, the function $f\left(\omega_{i} ; \Sigma, \gamma, \delta\right)$ can be interpreted as a "modified" risk contribution of asset $i$. Independently from the dispersion measure $D($.$) , solving the risk-based optimization program is equivalent to finding the portfolio$ allocation that satisfies

$$
\frac{\omega_{i}^{\gamma}}{\sigma_{i}^{\delta}} \times \frac{\partial \sigma_{p}}{\partial \omega_{i}}=\frac{\omega_{j}^{\gamma}}{\sigma_{j}^{\delta}} \times \frac{\partial \sigma_{p}}{\partial \omega_{j}} \quad \forall(i, j) \in\{1, \ldots, n\}
$$

with $\sum_{k=1}^{n} \omega_{k}=1$ and $\omega_{k} \geq 0, \forall k$. Given the values for $\delta$ and $\gamma$ (see Appendix A for more details), this optimization program encompasses the standard risk-based strategies such as MV, ERC, RP, and MD. Under mild assumptions on the covariance matrix $\Sigma$ (see Appendix B), the existence and unicity of the optimal risk-based portfolios are guaranteed.

One advantage of risk-based strategies is that they only depend on the portfolio risk, as measured by its volatility and ultimately by the covariance matrix $\Sigma$. In industry and academic literature, these strategies are generally based on the unconditional covariance matrix $\Sigma=\Sigma_{u}$. The optimal allocation $\omega_{u}^{*}$ then satisfies

$$
\omega_{u}^{*} \equiv \lambda\left(\Sigma_{u} ; \delta, \gamma\right)
$$

where $\lambda($.$) is a function { }^{4}$.

An alternative consists in using the conditional covariance matrix $\Sigma=\Sigma_{c, t} \equiv \mathbb{V}\left(r_{t} \mid \mathcal{F}_{t-h}\right)$ where $\mathcal{F}_{t-h}$ denotes the information set available at time $t-h$, for $h \geq 1$. The conditional approach allows to take into account the changes in economic environment and is generally more responsive to economic and financial news. In this case, the optimal allocation $\omega_{c, t}^{*}$ is defined by the same functional $\lambda($.$) , but is time-varying, with$

$$
\omega_{c, t}^{*} \equiv \lambda\left(\Sigma_{c, t} ; \delta, \gamma\right)
$$

The goal of our paper is to assess the advantages of the two approaches and to compare

\footnotetext{
${ }^{4}$ For most of cases, the functional $\lambda($.$) does not have a closed-form expression. The optimal portfolio is then$ the solution of a numerical optimization.
} 
the relative out-of-sample performances of the portfolios $\omega_{u}^{*}$ and $\omega_{c, t}^{*}$. To do so, we focus on 3 risk-based strategies, namely MV, ERC and MD. We now present these strategies in details. For ease of presentation, in what follows we do not make the distinction between conditional and unconditional approaches, and we denote by $\Sigma$ the covariance matrix.

\subsection{Minimum Variance}

Under the MV strategy, we choose the allocation that minimizes the variance of portfolio return. This strategy is the most popular among all the risk-based strategies. The MV portfolio, denoted $\omega^{M V}$, is the result of the following optimization program:

$$
\begin{gathered}
\omega^{M V}=\underset{\omega}{\arg \min } \omega^{\top} \Sigma \omega \\
\text { u.c. }\left\{\begin{array}{c}
e^{\top} \omega=1 \\
\omega \geq 0
\end{array} .\right.
\end{gathered}
$$

It is straightforward to show that the first order conditions (FOC) of the MV program (5) and the general risk-based allocation program (Equation 1) are equivalent for $\gamma=0$ and $\delta=0$. In our case, there is no closed-form solution for $\omega^{M V}$ and the optimal allocation has to be determined numerically. ${ }^{5}$ Since the MV portfolio minimizes the risk (measured by the volatility), it may induce a high concentration in the less risky assets.

\subsection{Equal Risk Contribution}

Contrary to the MV strategy, the ERC is a risk diversification strategy defined in terms of risk contributions. The first formal analysis of the ERC portfolio was given by Maillard et al. (2010), who establish its existence and uniqueness, derive a number of analytical properties, and propose numerical algorithms to compute the portfolio. The idea of ERC is to find a riskbalanced portfolio such that the risk contribution is the same for all assets of the portfolio. The risk contribution of asset $i$, denoted by $S_{i}(\omega, \Sigma)$, is defined as the share of total portfolio volatility attributable to that asset. Formally, we have

$$
S_{i}(\omega, \Sigma)=\frac{\omega_{i}}{\sigma_{p}} \times \frac{\partial \sigma_{p}}{\partial \omega_{i}}=\omega_{i} \frac{(\Sigma \omega)_{i}}{\omega^{\top} \Sigma \omega}
$$

where $(\Sigma \omega)_{i}$ is the $i^{t h}$ row of the $n$-dimensional vector $(\Sigma \omega)$. The risk contribution is expressed as a percentage of the portfolio volatility. Notice that the Euler's theorem implies $\sum_{i=1}^{n} S_{i}(\omega, \Sigma)=$

\footnotetext{
${ }^{5}$ However, when no constraint is imposed on the short-selling, the optimal allocation $\omega^{M V}$ has a closed-form expression given by

$$
\omega^{M V}=\lambda_{M V}(\Sigma ; 0,0)=\frac{\Sigma^{-1} e}{e^{\top} \Sigma^{-1} e} .
$$
}


1. The optimal ERC portfolio $\omega^{E R C}$ equalizes the risk contribution of all assets, such as

$$
S_{i}\left(\omega^{E R C}, \Sigma\right)=\frac{1}{n} \quad \forall i=1, \ldots, n
$$

This condition corresponds to the FOC of the general risk-based allocation program (Equation 1) with $\gamma=1$ and $\delta=0$.

Except under very restrictive conditions ( $n=2$ or equal correlations, see Roncalli (2014) for more details), there is no closed-form solution for $\omega^{E R C}$. In the general case, the ERC portfolio is the numerical solution of the following quadratic optimization program:

$$
\begin{gathered}
\omega^{E R C}=\underset{\omega}{\arg \min } \sum_{i=1}^{n} \sum_{j=1}^{n}\left(S_{i}(\omega, \Sigma)-S_{j}(\omega, \Sigma)\right)^{2} \\
\text { u.c. }\left\{\begin{array}{c}
e^{\top} \omega=1 \\
\omega \geq 0
\end{array} .\right.
\end{gathered}
$$

Maillard et al. (2010) show that the MV, ERC and Equally Weighted (EW) (i.e. the portfolio that equalizes the weights between assets) portfolio volatilities can be ranked in the following order $\sigma_{M V} \leq \sigma_{E R C} \leq \sigma_{E W}$. Hence, the ERC portfolio is naturally located between MV and EW and thus appears as a good potential substitute for these traditional approaches.

\subsection{Maximum Diversification}

The MD strategy has been introduced by Choueifaty and Coignard (2008). The optimal MD weights are determined in order to maximize the diversification ratio $D R(\omega ; \Sigma)$, defined as the portfolio's weighted average asset volatility to its actual volatility.

$$
D R(\omega ; \Sigma)=\frac{\omega^{\top} \sigma}{\left(\omega^{\top} \Sigma \omega\right)^{1 / 2}}
$$

with $\sigma=\left(\sigma_{1}, \ldots, \sigma_{n}\right)^{\top}$ the vector of individual volatilities. This ratio can be interpreted as the ratio between undiversified and diversified volatility of the portfolio (Choueifaty and Coignard (2008)). Formally, the optimal portfolio $\omega_{M D}^{*}$ is obtained by solving the following program:

$$
\begin{gathered}
\omega^{M D}=\underset{\omega}{\arg \max } \operatorname{DR}(\omega ; \Sigma) \\
\text { u.c. }\left\{\begin{array}{c}
e^{\top} \omega=1 \\
\omega \geq 0
\end{array}\right.
\end{gathered}
$$

Notice that the FOC of the MD program are equivalent to those of the general risk-based allocation program for $\gamma=0$ and $\delta=1$. 


\section{Benchmarking method}

In this section, we detail the implementation of the conditional and unconditional risk-based strategies and present the related estimation approaches. Then, we present our comparison framework and the criteria used to assess the out-of-sample performance of each strategy.

\subsection{Conditional and unconditional approaches}

In order to compare the conditional and unconditional approaches, we consider a sample $\left\{r_{1}, \ldots, r_{T}\right\}$ of historical asset returns. By definition, risk-based strategies depend on the covariance matrix of returns. ${ }^{6}$ Under the stationarity assumption for asset returns, the unconditional covariance matrix is constant over time, meaning that $\Sigma_{u}=\mathbb{V}\left(r_{t}\right), \forall t=1, \ldots, T$. As a consequence, the optimal portfolio weights $\omega_{u}^{*}=\lambda\left(\Sigma_{u} ; \delta, \gamma\right)$ are also constant for $t=1, \ldots, T$ and there is no turnover. This property clearly illustrates the main advantage of the unconditional approach for risk-based investment. However, such an approach assumes that the volatilities and correlations are constant whatever the economic and financial conditions (crisis period or not, news, etc.). On the contrary, the use of a conditional risk measure $\Sigma_{c, t} \equiv \mathbb{V}\left(r_{t} \mid \mathcal{F}_{t-h}\right)$ implies a positive turnover, since the corresponding optimal weights $\omega_{c, t}^{*}=\lambda\left(\Sigma_{c, t} ; \delta, \gamma\right)$ are time-varying with the information set $\mathcal{F}_{t-h}$. Thus, the conditional approach allows to incoporate the latest information in the optimal allocation. This is of great interest within a changing environment. For instance, Martellini, Milhau and Tarelli (2014) evoke the case of a risk parity strategy used for asset allocation in a context of low bond yield environment. This strategy will inevitably involve a substantial overweighting of bonds with respect to equities. Such an allocation may be problematic when a drop in long-term bond prices is likely to occur. Another difference between unconditional and conditional approaches, is that the latter necessarily implies the use of a model for the conditional covariance matrix, and hence raises the issue of misspecification error and model risk.

In practice, the covariance matrices are unobservable and have to be estimated. Assuming that returns are independently and identically distributed (i.i.d.), the unconditional covariance matrix can be estimated by its empirical counterpart, i.e. the sample covariance matrix of asset returns defined as

$$
\hat{\Sigma}_{u}=\frac{1}{T-1} \sum_{t=1}^{T}\left(r_{t}-\bar{r}\right)\left(r_{t}-\bar{r}\right)^{\top} .
$$

However, the i.i.d. assumption is likely to be violated given the stylized facts of the financial

\footnotetext{
${ }^{6}$ Other risk measures can be considered here. For instance, Martellini et al. (2014) define a new class of conditional risk parity portfolios with respect to downside risk measures such as semi-variance, Value-at-Risk (VaR) or Expected Shortfall.
} 


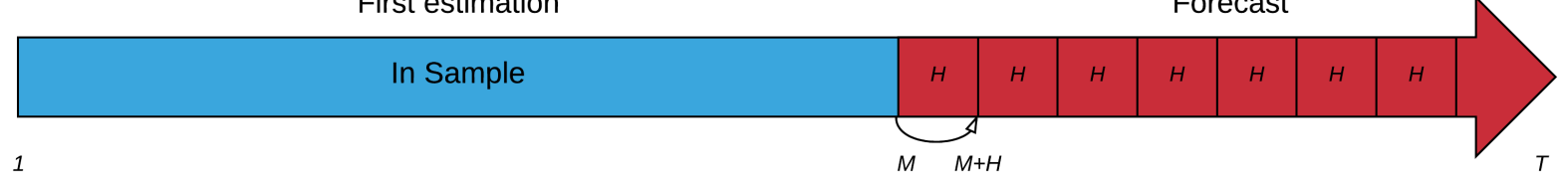

Second estimation

Forecast

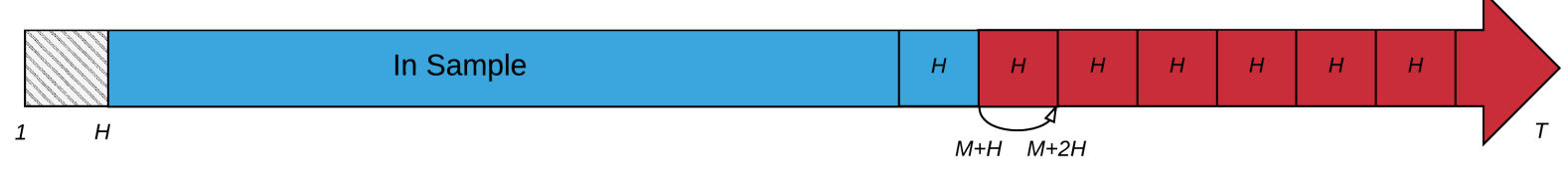

Figure 1: Rolling-window framework

time series. First, volatility of asset returns tends to change over time, and periods of high and low volatility tend to cluster together. Second, it is well known that in crisis periods the correlations between assets tend to sharply increase, reducing the diversification opportunities (Longin and Solnik (2001)). Besides, the sample covariance estimator gives the same weight to each of the past observations. To adress this issue, practitioners and academics regularly rebalance their portfolios and re-estimate the unconditional covariance matrix by using the most recent observations, with a rolling window estimation approach.

Let us assume that the investor rebalances his portfolio every $H$ periods and keeps the portfolio weights constant between two rebalancing dates. At each rebalancing date $s$, the investor forecasts the covariance matrix of the cumulated returns $R_{s+1: s+H}$ over the period $s+1$ to $s+H$. The out-of-sample forecast of the unconditional covariance matrix is simply defined as the sample covariance matrix of past asset returns. As usual in the literature, we consider a rolling-window estimator based on $M$ observations of the returns $r_{t}$, for $t=s-M, . ., s$. As displayed in Figure (1), the rebalancing dates are set at $s=M, M+H, M+2 H, \ldots, T-M-H$. For each rebalancing date $s$, the out-of-sample forecast of covariance matrix for the cumulated returns $R_{s+1: s+H}$ is given by

$$
\hat{\Sigma}_{u, s+1: s+H}=\frac{H}{M-1} \sum_{j=1}^{M}\left(r_{s-j+1}-\bar{r}_{s}\right)\left(r_{s-j+1}-\bar{r}_{s}\right)^{\top}, \forall s
$$

with $\bar{r}_{s}=M^{-1} \sum_{j=1}^{M} r_{s-j+1} .{ }^{7}$ Finally, the forecasted covariance matrix $\hat{\Sigma}_{u, s+1: s+H}$ is used to determine the optimal portfolio weights $\omega_{u, t}^{*}=\lambda\left(\hat{\Sigma}_{u, s+1: s+H} ; \delta, \gamma\right)$, which are fixed over the periods $t=s+1, \ldots, s+H$.

We proceed the same way for the conditional portfolios $\omega_{c, t}^{*}$. The only difference is that

\footnotetext{
${ }^{7}$ An alternative estimator for $\Sigma_{u, s+1: s+H}$ is given by the sample covariance matrix of the past cumulated returns. Here, we only consider the estimator given by Equation (12), which is based on the past daily returns, since its precision (i.e. the number of observations used to compute the sample covariance) does not change with the rebalancing horizon $H$.
} 
this approach implies the use of a dynamic model for the conditional covariance matrix $\Sigma_{c, s}=$ $\mathbb{V}\left(R_{s+1: s+H} \mid \mathcal{F}_{s}\right)$. Many alternative multivariate GARCH type-models can be considered here (see Bauwens et al. (2006) for a survey). Here, we consider the Dynamic Conditional Correlation (DCC) model introduced by Engle (2002) which is the most used in the literature. For ease of presentation, we consider the case $H=1$ and $R_{s+1: s+H}=r_{s+1}$. Formally, at each rebalancing date $s=t$ we assume that

$$
\Sigma_{c, t}=D_{t} C_{t} D_{t}
$$

where $C_{t}$ denotes the correlation matrix and $D_{t}$ is a diagonal matrix defined as

$$
D_{t}=\operatorname{diag}\left\{\sigma_{i, t}\right\}
$$

The conditional variance for the $i^{\text {th }}$ asset return, denoted $\sigma_{i, t}^{2}$, follows a univariate $\operatorname{GARCH}(1,1)$ process. Let $\varepsilon_{t}=D_{t}^{-1} r_{t}$ be the standardized returns and define $Q_{t}$ a symmetric positive definite matrix such that

$$
C_{t}=\left(\operatorname{diag}\left\{Q_{t}\right\}\right)^{-1 / 2} Q_{t}\left(\operatorname{diag}\left\{Q_{t}\right\}\right)^{-1 / 2} .
$$

The dynamics of $Q_{t}$ is given by the following GARCH-type expression:

$$
Q_{t}=\bar{Q}(1-\alpha-\beta)+\alpha\left(\varepsilon_{t-1} \varepsilon_{t-1}^{\top}\right)+\beta Q_{t-1}
$$

where $\bar{Q}$ is the unconditional correlation matrix of the standardized returns $\varepsilon_{t}, \alpha$ and $\beta$ are two positive parameters with $\alpha+\beta<1$. At each rebalancing date $s$, the model parameters $\theta=(\alpha, \beta)^{\top}$ are estimated by quasi-maximum likelihood (QML). As for the unconditional case, we consider a rolling window estimator based on $M$ past returns $r_{t}$ for $t=s-M, . ., s$. The conditional covariance matrix forecast $\widehat{\Sigma}_{c, s+1: s+H}=\widehat{\mathbb{V}}\left(R_{s+1: s+H} \mid \mathcal{F}_{s}\right)$ is then used to compute the optimal allocation $\omega_{c, t}^{*}=\lambda\left(\widehat{\Sigma}_{c, s+1: s+H} ; \delta, \gamma\right)$ for $t=s+1, \ldots, s+H{ }^{8}$

This process is repeated for the whole period. Finally, we get $T-M$ out-of-sample (ex-post) portfolio returns $\omega_{u, t}^{* \top} r_{t}$ and $\omega_{c, t}^{* T} r_{t}$, with $t=M, \ldots, T$, for both unconditional and conditional approaches of the risk-based strategies MV, ERC, and MD.

\subsection{Rebalancing frequency and sample size}

Because parameters $H$ (rebalancing frequency) and $M$ (estimation's sample size) are key inputs in our estimation procedure, we discuss how a change in their values may impact our results ex ante.

First, increasing the estimation window size $M$ diminishes the weight of recent information,

\footnotetext{
${ }^{8}$ Various methods can be used to compute the forecast $\widehat{\Sigma}_{c, s+1: s+H}$, including dynamic forecast, simulation, or approximation. As for the unconditional case, we consider the approximation $\widehat{\Sigma}_{c, s+1: s+H}=H \times \widehat{\mathbb{V}}\left(r_{s+1} \mid \mathcal{F}_{s}\right)$.
} 
and makes the covariance out-of-sample estimates less reactive to new information. At the limit, they tend to be constant over time and to converge to the true unconditional matrix. Under mild regularity assumptions, as soon as $M \rightarrow \infty$ with $M / n \rightarrow k>0$, we get for $H=1$ :

$$
\begin{aligned}
& \operatorname{plim} \hat{\Sigma}_{\substack{u, s+1 \\
M \rightarrow \infty}}=\Sigma_{u}=\mathbb{V}\left(r_{t}\right) \\
& \operatorname{plim} \hat{\Sigma}_{c, s+1}(\widehat{\theta})=\Sigma_{c, t+1}\left(\theta_{0}\right)=\mathbb{V}\left(r_{t} \mid \mathcal{F}_{t-1}\right)
\end{aligned}
$$

with $\theta_{0}$, the true value of the MGARCH model. The optimal unconditional portfolios tend to be time invariant, i.e. $\omega_{u, t}^{*}=\lambda\left(\hat{\Sigma}_{u, s+1} ; \delta, \gamma\right) \rightarrow \omega_{u}^{*} \lambda\left(\Sigma_{u} ; \delta, \gamma\right)$ as $M \rightarrow \infty$, since the new $H$ observations added at each rebalancing date do not change the information set used for the covariance matrix estimation. Obviously, this is not the case for the conditional portfolios, as $\mathbb{V}\left(r_{t} \mid \mathcal{F}_{t-1}\right)$ changes with information available to date. Consequently, over a long evaluation period with financial crises and recoveries, we would expect that the relative performances of the conditional portfolios will improve (relatively to those of the unconditional portfolios) as the sample size $M$ increases.

Turning to the rebalancing frequency $H$, its influence on the relative out-of-sample performances of the conditional approach is less clear. For simplicity, let us denote by $\hat{\Sigma}_{u, s+H}$ the covariance matrix of the daily (instead of cumulated) returns at horizon $H$. Consider the special case in which the sample size $M$ tends to infinity, implying that $\hat{\Sigma}_{c, s+H}(\widehat{\theta})$ converges to $\Sigma_{c, s+H}\left(\theta_{0}\right)$. In this case, when the rebalancing horizon $H$ tends to infinity, $\hat{\Sigma}_{u, s+H}$ converges to the unconditional covariance matrix, since under stationarity assumptions, we have

$$
\lim _{\substack{c, s+H \\ H \rightarrow \infty}}\left(\theta_{0}\right)=\Sigma_{u}
$$

Consequently, when $M$ and $H$ tend to infinity, we have

$$
\operatorname{plim} \hat{\Sigma}_{c, s+H}(\widehat{\theta})=p \operatorname{plim} \hat{\Sigma}_{H, M \rightarrow \infty} \hat{H}_{H, M \rightarrow \infty}=\Sigma_{u}
$$

Under these assumptions, the unconditional and conditional risk-based optimal portfolios tend to be equivalent, i.e. $\omega_{u, t}^{*} \rightarrow \omega_{c, t}^{*}$, as $H \rightarrow \infty$. Even though these results cannot be extended for finite sample sizes $M$, we would expect that the differences in the out-of-sample performances of both portfolios tend to decrease with $M$.

\subsection{Performance criteria}

The objective is to compare the out-of-sample performances of the unconditional and conditional approaches for each of the three risk-based strategies. For this purpose, we use the same performance criteria as in DeMiguel et al. (2009), namely (1) the Sharpe ratio, (2) the return-loss, 
(3) the turnover, and (4) the portfolio variance. The rest of this section briefly presents these criteria.

Formally, for each approach (conditional and unconditional), indexed by $z=\{u, c\}$, and each risk-based investment strategy, indexed by $g=\{M V, E R C, M D\}$, we have

$$
\begin{gathered}
\widehat{\mu}=\frac{1}{T-M} \sum_{t=1}^{T-M}\left(\omega_{z, t}^{g}\right)^{\top} r_{t} \\
\widehat{\sigma}^{2}=\frac{1}{T-M-1} \sum_{t=1}^{T-M}\left(\left(\omega_{z, t}^{g}\right)^{\top} r_{t}-\widehat{\mu}\right)^{2}
\end{gathered}
$$

For ease of presentation, we do not index the returns and the standard deviation for each strategy and each approach.

The out-of-sample Sharpe ratio (SR thereafter) represents the expected return by unit of risk and is computed as

$$
S R=\frac{\hat{\mu}}{\hat{\sigma}}
$$

with $\hat{\mu}$ the empirical mean and $\hat{\sigma}$ the standard deviation of the (ex-post) returns of the risk-based portfolio. For each risk-based investment strategy, we also compute the $p$-value associated to the test of the null hypothesis of no-difference between the Sharpe ratios of unconditional and conditional approaches. To do so, we use the test methodology used by DeMiguel et al. (2009), initialy introduced by Jobson and Korkie (1981) with the correction proposed by Memmel (2003). Formally, given two portfolios $i$ and $j$, with $\hat{\mu}_{i}, \hat{\mu}_{j}, \hat{\sigma}_{i}, \hat{\sigma}_{j}$ and $\hat{\sigma}_{i, j}$ their estimated mean, variance and covariance over a sample of size $T-M$, the test of the hypothesis $H_{0}: \hat{\mu}_{i} / \hat{\sigma}_{i}-\hat{\mu}_{j} / \hat{\sigma}_{j}=0$ is obtained by the following test statistic, which is asymptotically distributed as a standard normal:

$$
\hat{z}=\frac{\hat{\sigma}_{j} \hat{\mu}_{i}-\hat{\sigma}_{i} \hat{\mu}_{j}}{\sqrt{\hat{\theta}}}, \text { with } \hat{\theta}=\frac{1}{T-M}\left(2 \hat{\sigma}_{i}^{2} \hat{\sigma}_{j}^{2}-2 \hat{\sigma}_{i} \hat{\sigma}_{j} \hat{\sigma}_{i, j}+\frac{1}{2} \hat{\mu}_{i}^{2} \hat{\sigma}_{j}^{2}+\frac{1}{2} \hat{\mu}_{j}^{2} \hat{\sigma}_{i}^{2}-\frac{\hat{\mu}_{i} \hat{\mu}_{j}}{\hat{\sigma}_{i} \hat{\sigma}_{j}} \hat{\sigma}_{i, j}^{2}\right) .
$$

Second, we consider the return-loss (RL thereafter) of the unconditional approach with respect to the conditional approach. This criterion represents the additional return needed for the unconditional approach to perform as well as the conditional one. The RL is defined as

$$
R L=\frac{\hat{\mu}_{c}}{\hat{\sigma}_{c}} \times \hat{\sigma}_{u}-\hat{\mu}_{u}
$$

with $\hat{\mu}_{c}, \hat{\sigma}_{c}, \hat{\mu}_{u}$ and $\hat{\sigma}_{u}$ respectively denote the empirical mean and standard deviation of the conditional and unconditional approaches (ex-post) returns.

Then, we compare the turnover of the conditional and unconditional approaches. For that, 
many indicators can be used. Here, we measure the average turnover by

$$
\text { Turnover }=\frac{1}{S} \sum_{s=1}^{S} \sum_{i=1}^{n}\left|\omega_{i, M+(s+1) H}-\omega_{i, M+S H}\right|
$$

with $\omega_{i, s}$ the weight of $i^{\text {th }}$ asset at rebalancing date $s$ and $S=(T-M-H) / H$ the total number of rebalancing dates.

Finally, in order to evaluate the ex-post portfolio risk, we compute the empirical variance of the ex-post returns. We compute the $p$-value of the difference of variances between the unconditional and conditional approaches. To do so, we conduct a Levene's test of equality of variances, which is less sensitive about the hypothesis of normality.

\section{Data}

In order to apply our benchmarking method, we consider 3 empirical datasets used by DeMiguel et al. (2009) to compare the out-of-sample performance of the naive portfolio to the samplebased mean-variance model. We extend their datasets to the end of 2016. Notice that DeMiguel et al. (2009) consider monthly returns. Instead, we consider daily returns as it is well-known that it is the most favorable sampling frequency to identify conditional heteroskedasticity, i.e. $\mathrm{ARCH}$ effects. Hence, we consider the most favorable framework to identify the gains of the conditional risk-based investment strategies.

These 3 datasets include various number of assets and types of exposure: (1) Industry, (2) International and (3) Size and Book-to-Market. The portfolios included in these 3 datasets are exactly similar to those considered by DeMiguel et al. (2009). The value-weighted portfolios are constructed at the end of each June using the June market equity and NYSE breakpoints. A value weighted portfolio computes assets' weight based on its absolute and relative value as compared to other stocks in the portfolio.

Industry. The dataset "Industry" is composed of daily value-weighted returns on 10 industries $(n=10)$ in the United Sates. Formally, each NYSE, AMEX and NASDAQ stock is assigned to an industry. Industries considered are: Consumer-Discretionary, Consumer-Staples, Manufacturing, Energy, High-Technology, Telecommunication, Wholesale and Retail, Health, Utilities and Others. The portfolios are constructed at the end of June of each year. The portfolios' daily returns from January 2, 1996 to December 30, 2016 are taken from Kenneth French's website.

International. The dataset "International" contains $n=8$ international equity indices from MSCI (Morgan Stanley Capital International) for Canada, France, Germany, Italy, Japan, 
Switzerland, the UK and the US. Data range from June 1, 1994 to October 6, 2016.

Size and Book-to-Market. The SMB dataset is composed of twenty porfolios $(n=20)$ sorted by size and book-to-market ratio. As in DeMiguel et al. (2009) we exclude the five portfolios containing the largest firms. This results in the intersection of 4 portfolios formed on size (market equity) and 5 portfolios formed on the book-to-market ratio (book equity over market equity). Portfolios are constructed at the end of each June. Data from January 2, 1996 to December 30, 2016 are taken from Kenneth French's website.

\section{$5 \quad$ Empirical results}

In this section, we compare the different criteria for each risk-based strategy on unconditional and conditional frameworks and for each database. We also consider the naive strategy (or equally weighted portfolio) as a benchmark. This strategy involves holding a portfolio weight $\omega=1 / n$ in each of the $n$ assets (DeMiguel et al., 2009). For each strategy, we consider different rebalancing frequencies: $H=1$ for daily, $H=5$ for weekly, and $H=22$ for monthly rebalancing, respectively. We also consider two rolling window sizes, $M=500$ and $M=1,000$, in order to assess the influence of the estimation's sample size on the out-of-sample performances of the portfolios.

\section{Insert Table 1}

Table 1 reports the results for the out-of-sample SR for each rebalancing frequency $H$ and sample size $M$, for the 3 datasets. It also displays the $p$-value of the difference between conditional and unconditional portfolios. P-values in bold indicate significant differences between SRs. The main takeaway is that the conditional approach does not improve the performance of the portfolio in terms of SR and even worse, it can deteriorate it. Most of the SR differences are not significant at the 5 or $10 \%$ level. Furthermore, within the rare cases for which the differences are significant, the SRs of the unconditional strategies are higher than those of the conditional ones. For instance, for the portfolio 'International' with $M=500$, the SR differences observed for all the strategies are significant for $H=1$, but in these cases, the SRs of the unconditional strategies are largely higher than those of the conditional ones: 0.0285 versus 0.0168 for the MV strategy, 0.0239 versus 0.0206 for the MD strategy. As expected, the SRs of the unconditional approaches are stable with the rebalancing frequency $H$, but we observe a small improvement for the SRs of the conditional strategies. Hence, the performance differences of both approaches tend to decrease with $H$. However, as expected, the difference between the SRs of the two 
approaches increases globally with the sample size $M$. For instance, the SR difference between the unconditional and conditional RP of the 'Size' portfolio for $H=1$, becomes significant for $M=1,000$, while it was not in the case with $M=500$. Notice that this is the only case where the conditional performs better than the unconditional approach. We can also notice that SRs of both approaches improves with $M$.

\section{Insert Table 2}

Table 2 shows the results for the return-loss (RL), i.e. the additional return needed for the unconditional approach to perform as well as the conditional one. Recall that when RL is negative, the unconditional approach performs better than the conditional one. We report the results for each rebalancing frequency $H$ and sample size $M$. The conclusions here confirm those obtained with the SR. Our main result is that, whether the RL positive or negative, the gains or losses in terms of returns are negligible. In fact, whatever the portfolio, the rebalancing frequency, and the estimation window size, the RL is always close to 0. For the portfolio 'Size', the conditional approach dominates the unconditional approach, whereas the unconditional seems to be better for the portfolio 'International'. The RL is generally decreasing with $M$. For instance, for the portfolio 'Size', with $H=22$ for the MV strategy, the RL is going from 0.0033 with $M=500$ to -0.0041 with $M=1000$, many positive RL with $M=500$ become negative when $M=1000$. Finally, there is no clear-cut conclusion as regards the relationship between the rebalancing horizon $H$ and the out-of-sample gains in terms of returns.

\section{Insert Table 3}

As expected, the unconditional approach has a lower turnover than the conditional one. Table 3 reports the turnover for each strategy, each rebalancing date and each $M$. We also display the difference between unconditional and conditional turnovers. We can observe that the turnover of the conditional approach is always higher than that of the unconditional one. For all the strategies, the turnover increases sharply with $H$ for the conditional approach, going for instance from 0.3743 for daily rebalancing to 1.1465 for monthly rebalancing for the MV strategy applied to the portfolio 'Size', with $M=500$. The turnover of the unconditional approach is logically more stable with $H$, which leads to a decreasing gap. Then, increasing the rebalancing horizon $H$ benefits to the unconditional approach, while the estimation sample size $M$ shows no effect on the gaps between conditional and unconditional turnovers but a general reduction of both. 
Table 4 reports the empirical variance of the out-of-sample portfolio returns. For each strategy, we also display the p-value of the difference between the variances of the unconditional and conditional portfolios based on the Levene's test. For all the portfolios and investment strategies, the differences between the variances of the conditional and unconditional approaches are never statistically different. Despite the higher reactiveness of the conditional portfolios, the gain in terms of ex-post variance is negligible. In other words, we conclude that the conditional approach for risk-based investment doesn't reduce the ex-post portfolio risk.

To sum up, the implementation of a conditional approach shows no improvement of the risk dimension, while the performance is not enhanced and the turnover is largely increased. These negative results are robust to the type of assets and portfolios considered, the rebalancing horizon, and the estimation window size considered.

\section{Robustness check}

In this section, we analyse the robustness of our results to (i) the choice of the multivariate GARCH model used for the conditional approach and to (ii) the business cycles.

\subsection{Robustness to the conditional risk model}

We apply the same methodology as in Section 3, but we consider the Constant Conditional Correlation (CCC) model of Bollerslev (1990). The CCC model belongs to the same type of dynamic model as the DCC. The only difference relates to the correlation matrix, which is supposed constant in the CCC model, whereas it is assumed to be time-varying within the DCC. Formally, at each rebalancing date, the covariance matrix of the CCC model $\tilde{\Sigma}_{c, t}$ is defined as:

$$
\tilde{\Sigma}_{c, t}=D_{t} \bar{Q} D_{t}
$$

with $\bar{Q}$ the unconditional correlation matrix of returns defined in Section 3. Imposing a constant correlation matrix means that the only difference between the conditional and unconditional riskbased investment strategies comes from the volatility forecasts used to predict the individual risks of the assets.

The results are reported in Table 5 for $M=500$. Note that we conduct our methodology comparison for each of the 3 databases, and we report for each case the Sharpe ratios, the turnovers, and the variance. We consider the cases of daily $(H=1)$ and monthly $(H=22)$ rebalancing frequencies for comparison purpose. The results are similar to the ones with the 
DCC model. As for the DCC model, the Sharpe ratios are globally identical for conditional and unconditional approaches, as the differences are not significant. When the differences are significant, as for the portfolio "International" with $H=1$ for instance, the Sharpe ratio of the unconditional risk-based portfolio is higher than the for the conditional one. We also observe the higher turnover of the conditional approach. However, the conditional approach brings no improvement in terms of ex-post risk. The difference between the ex-post variances of conditional and unconditional portfolios are generally not significant. The only exception is for the portfolio "Industry" and the MD strategy. In this case, the conditional approach performs better in terms of ex-post risk.

\section{Insert Tables 5}

\subsection{Conditional versus unconditional approaches within business cycles}

We now analyse the relative performances of the two approaches depending on the economic conditions. Indeed, we could expect that the conditional risk-based portfolios perform better in period of crisis that unconditional risk-based portfolios. In order to assess the robustness of our negative results to the business cycles, we define two subsamples, one associated with crisis periods (i.e. periods with high volatility) and another sample of calm periods. For the crisis sample, we consider data from January 3, 2000 to December 31, 2001 and from January 2, 2008 to December 31, 2009; and we choose January 2, 2004 to December 30, 2005 and January 2, 2013 to December 31, 2014 for the calm periods. Results for $H=1$ and $M=500$ are reported in Table $6 .^{9}$

During the crisis periods, the differences between the SR of conditional and unconditional portfolios are significant. Surprisingly, the unconditional RP, MV or MD exhibit a larger SR than their conditional benchmarks for 2 portfolios out of 3 . For instance, for the MV strategy and the "Industry" dataset, the SR goes from 0.0186 with the unconditional approach to 0.0077 with the conditional approach. The only exception is the dataset "Size" for which the conditional approach seems to improve the performance of the risk-based portfolios. For instance, the SR is equal to 0.0211 for the unconditional MV strategy whereas it is equal to 0.0277 for the conditional approach. During the calm periods, the differences of SRs are globally not significant or in favor of the unconditional approach. Note that in some cases, the SR of the naive strategy (EW) is higher than that of the conditional approach for risk-based strategies. For instance, for the portfolio "Size", the naive SR is 0.0130 while it is 0.0100 for the conditional MD strategy. Concerning the ex-post risk, the conditional approach does not bring any improvement whatever

\footnotetext{
${ }^{9}$ Further results are available upon request.
} 
the business cycle. During the crisis and calm periods, the ex-post variances of the conditional and unconditional portfolios are very close and the differences are not significant. We only observe one case where the difference is significant: for calm periods and the MV strategy.

\section{Insert Table 6}

\section{Conclusion}

It is a well established fact that negative scientifc results may be relevant both for the academic community and industry (Harvey, 2017). We think that is the case for our study. In this paper, we propose the first systematic comparison of unconditional and conditional risk-based investment strategies. Our conclusion is clear: using a conditional risk measure does not improve the performance of the risk-based investment strategies. It does not improve the performance, it does not reduce the ex-post risk, but it increases the portfolio turnover. This result suggests that fund managers as well as asset managers should continue to give priority to model free approaches based on unconditional moments estimated on a rolling-window basis.

These conclusions are drawn from a comparison study based on 3 empirical datasets, 3 riskbased strategies (namely the MV, MD and ERC), various rebalancing horizons, and different estimation window lengths. They are robust to the use of different multivariate GARCH models to estimate the conditional covariance matrix. Our conclusions are also robust to the influence of business cycles: the conditional approach does not exhibit better performances than the unconditional one, even during crisis periods.

A natural extension of our work consists in using of Monte Carlo simulations. Using a theoretical framework with conditional heteroskedasticity and/or time-varying correlations for the asset returns, we could derive the optimal conditional portfolios for different risk-based strategies (MV, MD, ERC, etc.). By using Monte Carlo simulations for the returns, we could compute the estimates of the unconditional covariance matrix and determine the corresponding unconditional portfolios. By comparing both, we could asses the losses due to neglecting the heteroskedasticity and/or the time-varying property of correlations.

\section{References}

Ardia, D., Bolliger, G., Boudt, K., and Gagnon-Fleury, J.-P. (2017). The impact of covariance misspecification in risk-based portfolios. Annals of Operations Research, 254(1-2):1-16.

Bauwens, L., Laurent, S., and Rombouts, J. V. (2006). Multivariate garch models: a survey. Journal of applied econometrics, 21(1):79-109. 
Bollerslev, T. (1990). Modelling the coherence in short-run nominal exchange rates: a multivariate generalized arch model. The review of economics and statistics, pages 498-505.

Chopra, V. K. and Ziemba, W. T. (1993). The effect of errors in means, variances, and covariances on optimal portfolio choice. The Journal of Portfolio Management, 19(2):6-11.

Choueifaty, Y. and Coignard, Y. (2008). Toward maximum diversification. The Journal of Portfolio Management, 35(1):40-51.

Choueifaty, Y., Froidure, T., and Reynier, J. (2013). Properties of the most diversified portfolio. Journal of Investment Strategies, (2):49-70.

Chow, T.-m., Hsu, J., Kalesnik, V., and Little, B. (2011). A survey of alternative equity index strategies. Financial Analysts Journal, 67(5):37-57.

DeMiguel, V., Garlappi, L., and Uppal, R. (2009). Optimal versus naive diversification: How inefficient is the 1/n portfolio strategy? The Review of Financial Studies, 22(5):1915.

Engle, R. (2002). Dynamic conditional correlation: A simple class of multivariate generalized autoregressive conditional heteroskedasticity models. Journal of Business $\&$ Economic Statistics, 20(3):339-350.

Frankfurter, G. M., Phillips, H. E., and Seagle, J. P. (1971). Portfolio selection: the effects of uncertain means, variances, and covariances. Journal of Financial and Quantitative Analysis, 6(5):1251-1262.

Harvey, C. R. (2017). The scientific outlook in financial economics.

Jobson, J. D. and Korkie, B. M. (1981). Performance hypothesis testing with the sharpe and treynor measures. The Journal of Finance, 36(4):889-908.

Jurczenko, E., Michel, T., and Teiletche, J. (2013). Generalized risk-based investing.

Kan, R. and Zhou, G. (2007). Optimal portfolio choice with parameter uncertainty. Journal of Financial and Quantitative Analysis, 42(3):621-656.

Kolanovic, M., Kaplan, B., and Mehra, A. (2015). Technical selling: How low can it push equities? J.P. Morgan market and volatility commentary note.

Ledoit, O. and Wolf, M. (2004). Honey, i shrunk the sample covariance matrix. The Journal of Portfolio Management, 30(4):110-119. 
Ledoit, O. and Wolf, M. (2012). Nonlinear shrinkage estimation of large-dimensional covariance matrices. Annals of Statistics, 40(2):1024-1060.

Leote de Carvalho, R., Lu, X., and Moulin, P. (2012). Demystifying equity risk-based strategies: A simple alpha plus beta description. The Journal of Portfolio Management, 38(3):56-70.

Maillard, S., Roncalli, T., and Teïletche, J. (2010). The properties of equally weighted risk contribution portfolios. The Journal of Portfolio Management, 36(4):60-70.

Martellini, L., Milhau, V., and Tarelli, A. (2014). Hedging inflation-linked liabilities without inflation-linked instruments through long/short investments in nominal bonds. The Journal of Fixed Income, 24(3):5-29.

Memmel, C. (2003). Performance hypothesis testing with the sharpe ratio.

Merton, R. C. (1980). On estimating the expected return on the market: An exploratory investigation. Journal of financial economics, 8(4):323-361.

Michaud, R. O. (1989). The markowitz optimization enigma: Is 'optimized'optimal? Financial Analysts Journal, 45(1):31-42.

Qian, E. (2005). Risk parity portfolios: Efficient portfolios through true diversification. Panagora Asset Management.

Roncalli, T. (2013). Introduction to risk parity and budgeting. CRC Press.

Sender, H. and Wigglesworth, R. (2015). Investing: Whatever the weather? Financial Times. https://www.ft.com/content/54680182-46a1-11e5-b3b2-1672f710807b\#axzz46zmGfQqn. 
Table 1: Sharpe Ratio

\begin{tabular}{|c|c|c|c|c|c|c|c|c|c|}
\hline \multicolumn{10}{|c|}{ Sharpe Ratio $\mathrm{M}=500$} \\
\hline & \multicolumn{3}{|c|}{ Industry } & \multicolumn{3}{|c|}{ International } & \multicolumn{3}{|c|}{ Size } \\
\hline & $\mathrm{H}=1$ & $\mathrm{H}=5$ & $\mathrm{H}=22$ & $\mathrm{H}=1$ & $\mathrm{H}=5$ & $\mathrm{H}=22$ & $\mathrm{H}=1$ & $\mathrm{H}=5$ & $\mathrm{H}=22$ \\
\hline Uncond RP & 0.0354 & 0.0355 & 0.0356 & 0.0227 & 0.0227 & 0.0229 & 0.0366 & 0.0368 & 0.0370 \\
\hline Cond RP & 0.0350 & 0.0353 & 0.0357 & 0.0201 & 0.0220 & 0.0222 & 0.0371 & 0.0370 & 0.0374 \\
\hline P-value & 0.5780 & 0.8906 & 0.9614 & 0.0512 & 0.7948 & 0.8965 & 0.3022 & 0.8398 & 0.8181 \\
\hline Uncond MV & 0.0431 & 0.0433 & 0.0429 & 0.0285 & 0.0291 & 0.0293 & 0.0466 & 0.0466 & 0.0464 \\
\hline Cond MV & 0.0430 & 0.0436 & 0.0423 & 0.0168 & 0.0226 & 0.0209 & 0.0534 & 0.0487 & 0.0492 \\
\hline P-value & 0.9860 & 0.9164 & 0.9678 & 0.0005 & 0.3737 & 0.5776 & 0.0596 & 0.9310 & 0.8990 \\
\hline Uncond MD & 0.0344 & 0.0346 & 0.0347 & 0.0239 & 0.0239 & 0.0238 & 0.0313 & 0.0314 & 0.0319 \\
\hline Cond MD & 0.0348 & 0.0350 & 0.0348 & 0.0206 & 0.0222 & 0.0231 & 0.0320 & 0.0315 & 0.0321 \\
\hline P-value & 0.4577 & 0.7165 & 0.8467 & 0.0169 & 0.5275 & 0.8599 & 0.8732 & 0.7899 & 0.9399 \\
\hline EW & 0.0335 & 0.0336 & 0.0336 & 0.0209 & 0.0208 & 0.0211 & 0.0347 & 0.0349 & 0.0350 \\
\hline \multicolumn{10}{|c|}{ Sharpe Ratio $M=1,000$} \\
\hline & \multicolumn{3}{|c|}{ Industry } & \multicolumn{3}{|c|}{ International } & \multicolumn{3}{|c|}{ Size } \\
\hline & $\mathrm{H}=1$ & $\mathrm{H}=5$ & $\mathrm{H}=22$ & $\mathrm{H}=1$ & $\mathrm{H}=5$ & $\mathrm{H}=22$ & $\mathrm{H}=1$ & $\mathrm{H}=5$ & $\mathrm{H}=22$ \\
\hline Uncond RP & 0.0325 & 0.0327 & 0.0320 & 0.0168 & 0.0168 & 0.0165 & 0.0367 & 0.0367 & 0.0360 \\
\hline Cond RP & 0.0320 & 0.0319 & 0.0309 & 0.0137 & 0.0154 & 0.0157 & 0.0371 & 0.0373 & 0.0364 \\
\hline P-value & 0.5323 & 0.6556 & 0.7766 & 0.0352 & 0.6539 & 0.8887 & 0.6582 & 0.5432 & 0.8669 \\
\hline Uncond MV & 0.0443 & 0.0444 & 0.0436 & 0.0238 & 0.0242 & 0.0239 & 0.0478 & 0.0478 & 0.0469 \\
\hline Cond MV & 0.0419 & 0.0413 & 0.0410 & 0.0098 & 0.0145 & 0.0153 & 0.0466 & 0.0474 & 0.0435 \\
\hline P-value & 0.3600 & 0.6164 & 0.8426 & 0.0003 & 0.2495 & 0.5825 & 0.7329 & 0.6597 & 0.7224 \\
\hline Uncond MD & 0.0322 & 0.0324 & 0.0317 & 0.0188 & 0.0189 & 0.0184 & 0.0295 & 0.0295 & 0.0288 \\
\hline Cond MD & 0.0319 & 0.0313 & 0.0311 & 0.0144 & 0.0162 & 0.0173 & 0.0321 & 0.0302 & 0.0295 \\
\hline P-value & 0.2645 & 0.4749 & 0.7830 & 0.0072 & 0.4358 & 0.8386 & 0.5189 & 0.9842 & 0.9975 \\
\hline EW & 0.0297 & 0.0299 & 0.0292 & 0.0154 & 0.0152 & 0.0150 & 0.0349 & 0.0349 & 0.0342 \\
\hline
\end{tabular}

For each empirical dataset, this table reports the Sharpe ratio for each strategy for the conditional and unconditional approach and for each $H$ and $M$. It also displays the p-value of the difference between unconditional and conditional Sharpe ratios. P-values are in bold when the difference is statistically significant. 
Table 2: Return-loss

\begin{tabular}{|c|c|c|c|c|c|c|c|c|c|}
\hline \multicolumn{10}{|c|}{ Return-loss $\mathrm{M}=500$} \\
\hline & \multicolumn{3}{|c|}{ Industry } & \multicolumn{3}{|c|}{ International } & \multicolumn{3}{|c|}{ Size } \\
\hline & $\mathrm{H}=1$ & $\mathrm{H}=5$ & $\mathrm{H}=22$ & $\mathrm{H}=1$ & $\mathrm{H}=5$ & $\mathrm{H}=22$ & $\mathrm{H}=1$ & $\mathrm{H}=5$ & $\mathrm{H}=22$ \\
\hline $\mathrm{RP}$ & -0.0004 & -0.0002 & 0.0002 & -0.0025 & -0.0008 & -0.0007 & 0.0006 & 0.0002 & 0.0005 \\
\hline MV & -0.0001 & 0.0002 & -0.0006 & -0.0100 & -0.0056 & -0.0072 & 0.0079 & 0.0025 & 0.0033 \\
\hline MD & 0.0005 & 0.0004 & 0.0001 & -0.0029 & -0.0015 & -0.0006 & 0.0010 & 0.0002 & 0.0004 \\
\hline \multicolumn{10}{|c|}{ Return-loss $\mathrm{M}=1,000$} \\
\hline & \multicolumn{3}{|c|}{ Industry } & \multicolumn{3}{|c|}{ International } & \multicolumn{3}{|c|}{ Size } \\
\hline & $\mathrm{H}=1$ & $\mathrm{H}=5$ & $\mathrm{H}=22$ & $\mathrm{H}=1$ & $\mathrm{H}=5$ & $\mathrm{H}=22$ & $\mathrm{H}=1$ & $\mathrm{H}=5$ & $\mathrm{H}=22$ \\
\hline $\mathrm{RP}$ & -0.0006 & -0.0009 & -0.0012 & -0.0032 & -0.0015 & -0.0009 & 0.0006 & 0.0008 & 0.0004 \\
\hline MV & -0.0022 & -0.0029 & -0.0024 & -0.0125 & -0.0086 & -0.0077 & -0.0014 & -0.0005 & -0.0041 \\
\hline MD & -0.0004 & -0.0012 & -0.0007 & -0.0041 & -0.0024 & -0.0010 & 0.0037 & 0.0011 & 0.0010 \\
\hline
\end{tabular}

For each empirical dataset, this table reports the return-loss for the unconditional approach and for each $H$ and $M$. The RL is in bold when the conditional approach performs better (i.e. when it is positive). 
Table 3: Turnover

\begin{tabular}{|c|c|c|c|c|c|c|c|c|c|}
\hline \multicolumn{10}{|c|}{ Turnover $\mathrm{M}=500$} \\
\hline & \multicolumn{3}{|c|}{ Industry } & \multicolumn{3}{|c|}{ International } & \multicolumn{3}{|c|}{ Size } \\
\hline & $\mathrm{H}=1$ & $\mathrm{H}=5$ & $\mathrm{H}=22$ & $\mathrm{H}=1$ & $\mathrm{H}=5$ & $\mathrm{H}=22$ & $\mathrm{H}=1$ & $\mathrm{H}=5$ & $\mathrm{H}=22$ \\
\hline Uncond RP & 0.0014 & 0.0039 & 0.0112 & 0.0019 & 0.0052 & 0.0143 & 0.0008 & 0.0026 & 0.0085 \\
\hline Cond RP & 0.0317 & 0.0670 & 0.1050 & 0.0348 & 0.0753 & 0.1249 & 0.0253 & 0.0545 & 0.0792 \\
\hline Difference & -0.0303 & -0.0631 & -0.0938 & -0.0329 & -0.0701 & -0.1106 & -0.0245 & -0.0519 & -0.0707 \\
\hline Uncond MV & 0.0100 & 0.0269 & 0.0716 & 0.0100 & 0.0268 & 0.0716 & 0.0163 & 0.0480 & 0.1431 \\
\hline Cond MV & 0.2296 & 0.4854 & 0.6846 & 0.1601 & 0.3502 & 0.5747 & 0.3743 & 0.7928 & 1.1465 \\
\hline Difference & -0.2196 & -0.4585 & -0.6130 & -0.1502 & -0.3234 & -0.5031 & -0.3580 & -0.7448 & -1.0033 \\
\hline Uncond MD & 0.0113 & 0.0310 & 0.0793 & 0.0090 & 0.0237 & 0.0549 & 0.0184 & 0.0497 & 0.1292 \\
\hline Cond MD & 0.0743 & 0.1630 & 0.2778 & 0.0579 & 0.1302 & 0.2257 & 0.0727 & 0.1727 & 0.3138 \\
\hline Difference & -0.0630 & -0.1320 & -0.1985 & -0.0489 & -0.1065 & -0.1708 & -0.0543 & -0.1230 & -0.1845 \\
\hline EW & 0.0000 & 0.0000 & 0.0000 & 0.0000 & 0.0000 & 0.0000 & 0.0000 & 0.0000 & 0.0000 \\
\hline \multicolumn{10}{|c|}{ Turnover $\mathrm{M}=1,000$} \\
\hline & \multicolumn{3}{|c|}{ Industry } & \multicolumn{3}{|c|}{ International } & \multicolumn{3}{|c|}{ Size } \\
\hline & $\mathrm{H}=1$ & $\mathrm{H}=5$ & $\mathrm{H}=22$ & $\mathrm{H}=1$ & $\mathrm{H}=5$ & $\mathrm{H}=22$ & $\mathrm{H}=1$ & $\mathrm{H}=5$ & $\mathrm{H}=22$ \\
\hline Uncond RP & 0.0007 & 0.0021 & 0.0063 & 0.0010 & 0.0027 & 0.0079 & 0.0014 & 0.0014 & 0.0049 \\
\hline Cond RP & 0.0278 & 0.0630 & 0.1041 & 0.0319 & 0.0720 & 0.1163 & 0.0790 & 0.0484 & 0.0766 \\
\hline Difference & -0.0271 & -0.0609 & -0.0978 & -0.0309 & -0.0694 & -0.1084 & -0.0775 & -0.0470 & -0.0717 \\
\hline Uncond MV & 0.0055 & 0.0150 & 0.0425 & 0.0052 & 0.0142 & 0.0401 & 0.0257 & 0.0257 & 0.0882 \\
\hline Cond MV & 0.2131 & 0.4815 & 0.6966 & 0.1447 & 0.3351 & 0.5341 & 0.9331 & 0.7273 & 1.0999 \\
\hline Difference & -0.2076 & -0.4665 & -0.6540 & -0.1395 & -0.3209 & -0.4940 & -0.9074 & -0.7016 & -1.0117 \\
\hline Uncond MD & 0.0062 & 0.0171 & 0.0448 & 0.0047 & 0.0125 & 0.0304 & 0.0290 & 0.0290 & 0.0814 \\
\hline Cond MD & 0.0716 & 0.1749 & 0.3224 & 0.0559 & 0.1334 & 0.2417 & 0.1983 & 0.1844 & 0.3657 \\
\hline Difference & -0.0654 & -0.1578 & -0.2777 & -0.0512 & -0.1209 & -0.2113 & -0.1694 & -0.1554 & -0.2843 \\
\hline EW & 0.0000 & 0.0000 & 0.0000 & 0.0000 & 0.0000 & 0.0000 & 0.0000 & 0.0000 & 0.0000 \\
\hline
\end{tabular}

For each empirical dataset, this table reports the turnover for each strategy for the conditional and unconditional approach and for each $H$ and $M$. It also displays the gap between unconditional and conditional turnovers. Gaps are in bold when the conditional approach performs better than the unconditional one (i.e. when the conditional turnover is smaller). 
Table 4: Ex-post variance

\begin{tabular}{|c|c|c|c|c|c|c|c|c|c|}
\hline \multicolumn{10}{|c|}{ Variance $\mathrm{M}=500$} \\
\hline & \multicolumn{3}{|c|}{ Industry } & \multicolumn{3}{|c|}{ International } & \multicolumn{3}{|c|}{ Size } \\
\hline & $\mathrm{H}=1$ & $\mathrm{H}=5$ & $\mathrm{H}=22$ & $\mathrm{H}=1$ & $\mathrm{H}=5$ & $\mathrm{H}=22$ & $\mathrm{H}=1$ & $\mathrm{H}=5$ & $\mathrm{H}=22$ \\
\hline Uncond RP & 1.2131 & 1.2140 & 1.2183 & 0.9931 & 0.9938 & 0.9973 & 1.7722 & 1.7731 & 1.7776 \\
\hline Cond RP & 1.1849 & 1.1874 & 1.1949 & 0.9542 & 0.9491 & 0.9602 & 1.7397 & 1.7415 & 1.7466 \\
\hline P-value & 0.7352 & 0.7507 & 0.7802 & 0.4797 & 0.4130 & 0.5014 & 0.7408 & 0.7482 & 0.7538 \\
\hline Uncond MV & 0.8086 & 0.8096 & 0.8151 & 0.7323 & 0.7331 & 0.7406 & 1.3469 & 1.3516 & 1.3688 \\
\hline Cond MV & 0.8147 & 0.8237 & 0.8209 & 0.7119 & 0.7026 & 0.7117 & 1.2522 & 1.2600 & 1.2999 \\
\hline P-value & 0.9148 & 0.8112 & 0.9199 & 0.5889 & 0.4208 & 0.4402 & 0.2628 & 0.2757 & 0.4394 \\
\hline Uncond MD & 1.2877 & 1.2898 & 1.2938 & 0.7985 & 0.7968 & 0.7991 & 1.8568 & 1.8594 & 1.8717 \\
\hline Cond MD & 1.1888 & 1.1919 & 1.2041 & 0.7651 & 0.7608 & 0.7674 & 1.7858 & 1.7917 & 1.8102 \\
\hline P-value & 0.2986 & 0.3071 & 0.3496 & 0.4110 & 0.3692 & 0.4289 & 0.5276 & 0.5485 & 0.5900 \\
\hline EW & 1.3488 & 1.3492 & 1.3518 & 1.1698 & 1.1710 & 1.1730 & 1.8437 & 1.8441 & 1.8472 \\
\hline \multicolumn{10}{|c|}{ Variance $M=1,000$} \\
\hline & \multicolumn{3}{|c|}{ Industry } & \multicolumn{3}{|c|}{ International } & \multicolumn{3}{|c|}{ Size } \\
\hline & $\mathrm{H}=1$ & $\mathrm{H}=5$ & $\mathrm{H}=22$ & $\mathrm{H}=1$ & $\mathrm{H}=5$ & $\mathrm{H}=22$ & $\mathrm{H}=1$ & $\mathrm{H}=5$ & $\mathrm{H}=22$ \\
\hline Uncond RP & 1.2572 & 1.2580 & 1.2632 & 1.0802 & 1.0812 & 1.0834 & 1.8960 & 1.8960 & 1.9024 \\
\hline Cond RP & 1.2215 & 1.2237 & 1.2290 & 1.0197 & 1.0155 & 1.0282 & 1.8548 & 1.8490 & 1.8621 \\
\hline $\mathrm{P}$-value & 0.6980 & 0.7102 & 0.7112 & 0.3244 & 0.2818 & 0.3735 & 0.7063 & 0.6666 & 0.7132 \\
\hline Uncond MV & 0.8693 & 0.8702 & 0.8781 & 0.7940 & 0.7950 & 0.8016 & 1.4912 & 1.4912 & 1.5043 \\
\hline Cond MV & 0.8453 & 0.8505 & 0.8616 & 0.7459 & 0.7372 & 0.7570 & 1.4168 & 1.3410 & 1.4164 \\
\hline P-value & 0.7077 & 0.7633 & 0.7986 & 0.2540 & 0.1680 & 0.3034 & 0.4328 & 0.1050 & 0.3692 \\
\hline Uncond MD & 1.3568 & 1.3585 & 1.3637 & 0.8441 & 0.8440 & 0.8489 & 2.0395 & 2.0395 & 2.0494 \\
\hline Cond MD & 1.2442 & 1.2473 & 1.2537 & 0.8026 & 0.8000 & 0.8158 & 1.9013 & 1.9339 & 1.9553 \\
\hline $\mathrm{P}$-value & 0.2815 & 0.2925 & 0.2940 & 0.3456 & 0.3139 & 0.4609 & 0.2750 & 0.4101 & 0.4681 \\
\hline EW & 1.3815 & 1.3819 & 1.3863 & 1.2526 & 1.2541 & 1.2544 & 1.9532 & 1.9532 & 1.9583 \\
\hline
\end{tabular}

For each empirical dataset, this table reports the variance for each strategy for the conditional and unconditional approach and for each $H$ and $M$. It also displays the p-value of the Levene's test of equality of variances. 
Table 5: Comparison of unconditional and conditional risk-based portfolios: the case of a CCC model with $M=500$

\begin{tabular}{|c|c|c|c|c|c|c|c|c|c|}
\hline \multicolumn{10}{|c|}{ CCC Model $\mathrm{H}=1 ; \mathrm{M}=500$} \\
\hline & \multicolumn{3}{|c|}{ Industry } & \multicolumn{3}{|c|}{ International } & \multicolumn{3}{|c|}{ Size } \\
\hline & $\mathrm{SR}$ & Turnover & Variance & SR & Turnover & Variance & SR & Turnover & Variance \\
\hline Uncond RP & 0.0354 & 0.0014 & 1.2131 & 0.0227 & 0.0019 & 0.9931 & 0.0366 & 0.0008 & 1.7722 \\
\hline Cond RP & 0.0353 & 0.0315 & 1.1759 & 0.0197 & 0.0336 & 0.9667 & 0.0369 & 0.0252 & 1.7453 \\
\hline P-value/Difference & 0.9522 & -0.0301 & 0.6550 & 0.0246 & -0.0316 & 0.6335 & 0.5611 & -0.0244 & 0.7846 \\
\hline Uncond MV & 0.0431 & 0.0100 & 0.8086 & 0.0285 & 0.0100 & 0.7323 & 0.0466 & 0.0163 & 1.3469 \\
\hline Cond MV & 0.0457 & 0.2164 & 0.8371 & 0.0150 & 0.1825 & 0.7340 & 0.0521 & 0.3730 & 1.2653 \\
\hline P-value/Difference & 0.2891 & -0.2064 & 0.6241 & 0.0006 & -0.1725 & 0.9670 & 0.1471 & -0.3567 & 0.3375 \\
\hline Uncond MD & 0.0344 & 0.0113 & 1.2877 & 0.0239 & 0.0090 & 0.7985 & 0.0313 & 0.0184 & 1.8568 \\
\hline Cond MD & 0.0363 & 0.0334 & 1.1045 & 0.0166 & 0.0347 & 0.8148 & 0.0308 & 0.0271 & 1.9083 \\
\hline P-value/Difference & 0.7669 & -0.0221 & 0.0458 & 0.0012 & -0.0257 & 0.6993 & 0.9788 & -0.0087 & 0.6504 \\
\hline EW & 0.0335 & 0.0000 & 1.3488 & 0.0209 & 0.0000 & 1.1698 & 0.0347 & 0.0000 & 1.8437 \\
\hline \multicolumn{10}{|c|}{ CCC Model $\mathrm{H}=22 ; \mathrm{M}=500$} \\
\hline & \multicolumn{3}{|c|}{ Industry } & \multicolumn{3}{|c|}{ International } & \multicolumn{3}{|c|}{ Size } \\
\hline & SR & Turnover & Variance & SR & Turnover & Variance & SR & Turnover & Variance \\
\hline Uncond RP & 0.0356 & 0.0112 & 1.2183 & 0.0229 & 0.0143 & 0.9973 & 0.0370 & 0.0085 & 1.7776 \\
\hline Cond RP & 0.0358 & 0.1029 & 1.1851 & 0.0216 & 0.1215 & 0.9688 & 0.0372 & 0.0788 & 1.7519 \\
\hline P-value/Difference & 0.9371 & -0.0917 & 0.6904 & 0.8215 & -0.1072 & 0.6076 & 0.8997 & -0.0703 & 0.7952 \\
\hline Uncond MV & 0.0429 & 0.0716 & 0.8151 & 0.0293 & 0.0716 & 0.7406 & 0.0464 & 0.1431 & 1.3688 \\
\hline Cond MV & 0.0436 & 0.6404 & 0.8301 & 0.0187 & 0.6403 & 0.7318 & 0.0505 & 1.1205 & 1.3161 \\
\hline P-value/Difference & 0.9392 & -0.5688 & 0.7943 & 0.5556 & -0.5687 & 0.8197 & 0.8024 & -0.9774 & 0.5558 \\
\hline Uncond MD & 0.0347 & 0.0793 & 1.2938 & 0.0238 & 0.0549 & 0.7991 & 0.0319 & 0.1292 & 1.8717 \\
\hline Cond MD & 0.0372 & 0.1085 & 1.1117 & 0.0191 & 0.1248 & 0.8201 & 0.0292 & 0.0888 & 1.9266 \\
\hline P-value/Difference & 0.9790 & -0.0292 & 0.0466 & 0.6577 & -0.0699 & 0.6153 & 0.6553 & 0.0404 & 0.6330 \\
\hline EW & 0.0336 & 0.0000 & 1.3518 & 0.0211 & 0.0000 & 1.1730 & 0.0350 & 0.0000 & 1.8472 \\
\hline
\end{tabular}

For each empirical dataset, this table reports the criteria for each strategy for the conditional and unconditional approach and for $H=1 ; 22$ and $M=500$. It also displays the gap/p-value between unconditional and conditional criteria. Gaps/p-values are in bold when the conditional approach performs better than the unconditional one or when the difference is statistically significant. 
Table 6: Sharpe ratio and ex-post variance in crisis and calm periods

\begin{tabular}{|c|c|c|c|c|c|c|}
\hline \multicolumn{7}{|c|}{ Crisis period $H=1 ; M=500$} \\
\hline & \multicolumn{2}{|c|}{ Industry } & \multicolumn{2}{|c|}{ International } & \multicolumn{2}{|c|}{ Size } \\
\hline & $\mathrm{SR}$ & Variance & $\mathrm{SR}$ & Variance & SR & Variance \\
\hline Uncond RP & 0.0009 & 2.5285 & -0.0411 & 2.0277 & 0.0161 & 3.6665 \\
\hline Cond RP & -0.0015 & 2.4348 & -0.0458 & 1.9731 & 0.0172 & 3.5597 \\
\hline P-value & 0.0018 & 0.7897 & 0.0000 & 0.8160 & 0.0519 & 0.7837 \\
\hline Uncond MV & 0.0186 & 1.5880 & -0.0483 & 1.4192 & 0.0211 & 2.8829 \\
\hline Cond MV & 0.0077 & 1.6127 & -0.0582 & 1.4828 & 0.0277 & 2.6424 \\
\hline P-value & 0.0026 & 0.9177 & 0.0203 & 0.6890 & 0.0508 & 0.4854 \\
\hline Uncond MD & 0.0065 & 2.9166 & -0.0526 & 1.5299 & 0.0078 & 4.1664 \\
\hline Cond MD & 0.0044 & 2.5595 & -0.0562 & 1.5244 & 0.0100 & 3.9200 \\
\hline P-value & 0.2769 & 0.3899 & 0.0552 & 0.9740 & 0.0516 & 0.5947 \\
\hline EW & -0.0025 & 2.8278 & -0.0374 & 2.3376 & 0.0130 & 3.8391 \\
\hline \multicolumn{7}{|c|}{ Calm period $\mathrm{H}=1 ; \mathrm{M}=500$} \\
\hline & \multicolumn{2}{|c|}{ Industry } & \multicolumn{2}{|c|}{ International } & \multicolumn{2}{|c|}{ Size } \\
\hline & $\mathrm{SR}$ & Variance & SR & Variance & $\mathrm{SR}$ & Variance \\
\hline Uncond RP & 0.0888 & 0.4635 & 0.0787 & 0.4040 & 0.0781 & 0.8154 \\
\hline Cond RP & 0.0882 & 0.4577 & 0.0771 & 0.4002 & 0.0794 & 0.8032 \\
\hline P-value & 0.4720 & 0.8659 & 0.3731 & 0.9199 & 0.0000 & 0.8251 \\
\hline Uncond MV & 0.0959 & 0.3791 & 0.0884 & 0.4005 & 0.0815 & 0.6616 \\
\hline Cond MV & 0.0957 & 0.3707 & 0.0806 & 0.3238 & 0.1049 & 0.6151 \\
\hline P-value & 0.9550 & 0.7744 & 0.2471 & 0.0289 & 0.0000 & 0.3134 \\
\hline Uncond MD & 0.0988 & 0.4859 & 0.0856 & 0.4200 & 0.0839 & 0.7638 \\
\hline Cond MD & 0.0954 & 0.4674 & 0.0897 & 0.3677 & 0.0821 & 0.7757 \\
\hline P-value & 0.0252 & 0.6056 & 0.1602 & 0.1744 & 0.0987 & 0.8282 \\
\hline EW & 0.0841 & 0.4911 & 0.0719 & 0.4581 & 0.0773 & 0.8321 \\
\hline
\end{tabular}

For each empirical dataset, this table reports the Sharpe ratio and the variance for each strategy for the conditional and unconditional approach for $H=1$ and $M=500$ in case of crisis and calm periods. It also displays the pvalues of the difference between conditional and unconditional Sharpe ratios and variances. P-values are in bold when the difference is statistically significant. 


\section{Appendix}

\section{A Risk-based generalized strategies}

Denote by $M R C_{i}$ the following expression:

$$
M R C_{i}=\frac{\partial \sigma_{p}}{\partial \omega_{i}}=\frac{(\Sigma \omega)_{i}}{\omega^{\top} \Sigma \omega},
$$

which represents the marginal risk contribution of asset $i$. The marginal risk contribution of asset $i$ can be seen as the sensitivity of the portfolio total risk to a small change in the weight of asset $i$. It is straightforward to find the first order condition of the optimization program (1):

$$
\frac{\omega_{i}^{\gamma}}{\sigma_{i}^{\delta}} \times M R C_{i}=\frac{\omega_{j}^{\gamma}}{\sigma_{j}^{\delta}} \times M R C_{j}=\tau \quad \forall(i, j)=1, \ldots, N
$$

with

$$
\sum_{i=1}^{N} \omega_{i}=1
$$

where $\tau$ is a positive target constraint which is not necessary known to solve the program.

In Table 7 , the way to obtain our different risk-based portfolios with respect to $\gamma$ and $\delta$ from the program (1) is reported.

Table 7: Risk-based strategies

\begin{tabular}{c|c|c}
\hline \hline Portfolio & $(\gamma, \delta)$ & Strategy definition \\
\hline \hline ERC & $(1,0)$ & $\omega_{i} M R C_{i}=\omega_{j} M R C_{j}$ \\
MV & $(0,0)$ & $M R C_{i}=M R C_{j}$ \\
MD & $(0,1)$ & $\sigma_{i}^{-1} M R C_{i}=\sigma_{j}^{-1} M R C_{j} \quad\left(\rho_{i p}=\rho_{j p}\right)$ \\
$1 / \mathrm{N}$ & $(\infty, 0)$ & $\omega_{i}=\omega_{j}=N^{-1}$
\end{tabular}

\section{B Existence and uniqueness}

In order to discuss the existence and uniqueness of the optimal risk-based portfolios, we use an alternative expression of the optimization program suggested by Jurczenko et al. (2013):

$$
\begin{aligned}
& \omega^{*}=\underset{\omega}{\arg \min } \frac{1}{2} \omega^{\top} \Sigma \omega \\
& \text { u.c. } \sum_{i=1}^{n} \frac{\sigma_{i}^{\delta}\left(\omega_{i}^{1-\gamma}-1\right)}{(1-\gamma)} \geq c,
\end{aligned}
$$

where $c$ is a constant that depends on the risk-based strategy. The associated Lagrangian function is:

$$
L(\omega ; \lambda)=\frac{1}{2} \omega^{\top} \Sigma \omega-\lambda\left\{\sum_{i=1}^{n} \frac{\sigma_{i}^{\delta}\left(\omega_{i}^{1-\gamma}-1\right)}{(1-\gamma)}-c\right\}
$$


whith $\lambda$ the associated Lagrange multiplier. The first order condition is then given by:

$$
\frac{\partial L(\omega ; \lambda)}{\partial \omega}=\Sigma \omega-\lambda \nu
$$

with $\nu=\left(\sigma_{1}^{\delta} / \omega_{1}^{\gamma} \ldots \sigma_{n}^{\delta} / \omega_{n}^{\gamma}\right)^{\top}$. For each asset, this condition can be rewritten as:

$$
\frac{\left(\omega_{i}^{*}\right)^{\gamma}}{\sigma_{i}^{\delta}} \times(\Sigma \omega)_{i}=\lambda \quad \forall i=1, \ldots, n
$$

where $(\Sigma \omega)_{i}$ denotes the $i^{\text {th }}$ row of the matrix $\Sigma \omega$.

Define $\sigma_{p}=\left(\omega^{\top} \Sigma \omega\right)^{1 / 2}$ the portfolio volatility and $\partial \sigma_{p} / \partial \omega_{i}=(\Sigma \omega)_{i} / \sigma_{p}$ the marginal risk contribution of asset $i$. Then, for each asset, the first order condition can be rewritten as:

$$
\frac{\left(\omega_{i}^{*}\right)^{\gamma}}{\sigma_{i}^{\delta}} \times \frac{\partial \sigma_{p}}{\partial \omega_{i}}=\frac{\lambda}{\sigma_{p}} \quad \forall i=1, \ldots, n
$$

The optimal solution leads to equalize the modified risk contributions. We see that the first order condition of (1) and (28) are the same, that proves the equivalence of the two optimization programs. The uniqueness of the solution is guaranteed by the convexity of the program. The computation of the second order conditions gives:

$$
\frac{\partial^{2} L(\omega ; \lambda)}{\partial \omega^{2}}=\Sigma+\gamma \lambda \kappa
$$

with $\kappa=\left(\sigma_{1}^{\delta} / \omega_{1}^{\gamma+1} \ldots \sigma_{n}^{\delta} / \omega_{n}^{\gamma+1}\right)^{\top}$. The solution exists and is unique as long as this expression is positive. As $\gamma$ and $\delta$ are positive parameters, the solution exists as long as $\Sigma$ is a definite-positive matrix. Under this assumption, all optimal risk-based portfolios considered in this paper (MV, ERC, MD, 1/N) will be uniquely defined. 